\title{
Convolution in human rods: an ageing process
}

\author{
JOHN MARSHALL, ${ }^{1}$ JOHN GRINDLE, ${ }^{2}$ PAUL L. ANSELL, ${ }^{1}$ AND \\ BESSIE BORWEIN ${ }^{3}$ \\ From the ${ }^{1}$ Department of Visual Science, Institute of Ophthalmology, London, England; the ${ }^{2}$ Department of \\ Clinical Ophthalmology, Institute of Ophthalmology and Moorfields Eye Hospital, London, England; and \\ the ${ }^{3}$ Department of Physics, University of Western Ontario, London, Canada
}

SUMMARY In a morphological survey of 73 human retinae spanning 9 decades, and including 20 retinae which were obtained from eyes enucleated for malignant melanomas, nodular excrescences were noted in the outer segments of rods with an incidence which increased with age. These structures were sectioned in both their vertical and horizontal axes and on electron microscopical examination were seen to result from the localised convolution of affected outer segments. The topographic morphology of such convolutions is described and their modes of formation are discussed.

The process of ageing and its manifestations in the cells of the human retina has for some time been a major research interest in our laboratory. These studies include the electron microscopical examination of 73 human retinae obtained from both enucleations and cadaver eyes. This series contains specimens from each of 9 decades, the youngest eye being 19 years and the oldest 90 years. The detailed morphology of the photoreceptor cells, the pigment epithelium, and Brüch's membrane have been investigated in order to establish control reference material for a future study of senile macular degenerations and inherited retinal dystrophies. We were interested therefore by a recent report purporting to show the fusion of the outer segments of juxtapositioned rod photoreceptor cells in the retina of a 47-year-old woman whose eye was enucleated because of the presence of a choroidal melanoma (Borwein et al., 1977). In the present paper morphological evidence shows that this suggested fusion of appositioned cells is an age-related process occurring within the outer segments of individual rods.

\section{Methods}

One of the commonest conditions that leads to enucleation of human eyes is malignant melanoma of the uveal tract. The retinae of 20 such eyes have been studied in the present survey, with an age

Address for reprints: Dr John Marshall, Department of Visual Science, Institute of Ophthalmology, Judd Street, London WC1H 9QS range spanning 7 decades. The immediate postoperative procedures varied with the individual demands of participating centres. In most centres corneal discs were trephined for subsequent use in graft procedures. One centre required the dissection of the globe and the hemisection of the unfixed tumour so that samples could be processed for tissue culture as well as histopathology. In some cases intact globes were presented immediately after enucleation, and here transcorneal incisions were made in the sagittal plain before immersion in fixative.

All eyes were fixed within minutes of enucleation by immersion in $100 \mathrm{ml}$ of $0.3 \mathrm{M}$ glutaraldehyde buffered in $0.1 \mathrm{M}$ sodium cacodylate containing $10 \mathrm{mg} / \mathrm{ml}$ of calcium chloride with a final $\mathrm{pH} 7 \cdot 4$. Intact globes were progressively dissected in this solution, the cornea being removed at the sulcus sclerae after 5 minutes, the iris after a further 5 minutes, and the lens, ciliary body, and vitreous 20 minutes after the initial immersion.

After a total of 1 hour in fixative all posterior eye cups were washed in $0.1 \mathrm{M}$ sodium cacodylate containing $7.5 \%$ sucrose, and in this solution experimental samples were isolated under a dissecting microscope. In all cases the tumour was isolated first and subsequently processed for conventional histopathology. Where the topography of the tumour allowed, 5 sets of samples were isolated for electron microscopical study. These were the macula and 4 bands of tissue radiating from the macula to the ora serrata in the cardinal axes. All samples were dissected so that their 
orientation with respect to the macula could be determined during subsequent ultramicrotomy, and their distance from this locus was recorded. The isolated specimens were postfixed for 1 hour in $2 \%$ osmium tetroxide in $0.2 \mathrm{M}$ sodium cacodylate before being dehydrated by progressively increasing concentrations of ethanol in water and embedded in Epon 812 via epoxypropane.

Cadaver eyes were processed as above, being progressively dissected in glutaraldehyde. Where possible, the time interval between death and enucleation was recorded, as was the time between enucleation and fixation.

All sample blocks were first mounted in a Cambridge Huxley ultramicrotome and $0 \cdot 8-\mu \mathrm{m}$ sections were cut with glass knives. Most blocks were cut in at least two planes, first along the axis of the photoreceptor outer segments, and secondly at right-angles to this axis. Thick sections were stained with either toluidine blue (Meek, 1963) or basic fuchsin and methylene blue (Huber et al., 1968). By means of a chuck adaptor orientated blocks were transferred to a Reichert OMU3 ultramicrotome where $400-\AA \AA(40-\mathrm{nm})$ sections were cut with a diamond knife. These sections for electron microscopy were mounted on 200-mesh copper grids and stained with uranyl acetate and lead citrate before being examined in an AEI 801 electron microscope.

Further samples, representative of each decade, were isolated after glutaraldehyde fixation, and these were frozen in isopentane precooled in liquid nitrogen before being mounted in a Slee cryostat. Frozen sections $10 \mu \mathrm{m}$ thick were examined under a Zeiss microscope fitted with an epifluorescence attachment to determine the relative autofluorescence due to lipofuscin in the retinal pigment epithelium.

\section{Results}

Extensive observations in the present study have shown that in eyes enucleated for choroidal melanoma the morphologies of areas of retinae remote from the tumour are indistingushable from comparable areas of retinae in eyes of similar age obtained from cadavers with no known ophthalmic disease. These observations are in agreement with previous studies (Borwein et al., 1977). In most cases the fixation of retinae in surgically enucleated eyes was superior to that achieved in post-mortem specimens. However, significant observations were made on both groups and statistical data were pooled.

When transverse sections of retinae in the second and third decades were examined under the light microscope, the outer segments of rod photoreceptor cells were seen as homogeneously staining elongated rectangular systems with regular parallel borders in their long axis. In samples from the central or perimacular regions such outer segments were approximately $1.4 \mu \mathrm{m}$ in diameter and between 24 and $27 \mu \mathrm{m}$ in length (Fig. 1). The discrete nature of these systems was apparent in sections cut along the axis of the outer segment and in those at right-angles to this axis, as in both cases a poorly stained interreceptor matrix was distinguished. In all sections the rod outer segments experienced an angular deviation between their ciliary region and the pigment epithelium, and the degree of inclination varied with retinal position, a finding common in other vertebrates (Laties and Enoch, 1977).

Changes in the morphology of rod outer segments began to appear in the fourth decade and occurred initially in the perimacular regions. In light microscopic sections such changes were seen as nodular swellings, distorting either the central region, or pigment epithelial third, of the rod outer segment profile (Fig. 1). The rod diameters were increased to between 2.5 and $3.5 \mu \mathrm{m}$ in the region of these distortions. With increasing age such distortions became apparent in all areas of retina and in an increasing number of rod cells. By the seventh decade between 10 and $20 \%$ of the total rod population was involved.

In the electron microscope, sections of rod outer segments of young eyes showed few deviations from the typical ordered membrane array presented by the stacks of lobed discs. When such deviations occurred, they usually presented as groups of 10 to 30 discs which had become disorientated with respect to both their fellows and the boundary membrane of the cell. In some instances several such dislocations of the disc array occurred within the outer segment of a single receptor cell. A further anomaly was the occasional presence of regions of small vesicles which occurred parallel to and within the plane of the disc membranes. One or both of these phenomena occurred in some receptor cells in every sample examined. However, they never involved the boundary membrane of the cell, whose parallel configuration was always preserved in these regions. Small dislocations of the disc stacks which involved the boundary membranes were occasionally observed in young eyes, but these were always at the tips of rods, where they were surrounded by the receptor sheaths of the pigment epithelium. These occurrences usually resulted from the presence of disorganised or shed discs prior to their being phagocytosed.

In the rods of the older eyes the nodular expansions were identified as areas in which the outer segments had corrugated within their long axes 
Fig. 1 (a) Photomicrograph of a transverse section showing the receptor epithelial junction in the perimacular region of the retina of a 19-year-old male. The boundary membranes of the rods are parallel with no excrescences. Brüch's membrane is thin and the pigment epithelium contains little lipofuscin.

(b) Photomicrograph of a tangential section through the receptor outer segments in the retina seen in (a) and demonstrating the isolation of appositioned elements.

(c) Photomicrograph of a transverse section through the receptor epithelial junction in the perimacular region of the retina of a 54-year-old female. Nodular excrescences can be seen associated with several rod outer segments. Brüch's membrane is thicker than in (a) and an increased lipofuscin content is apparent.

(d) Photomicrograph of a tangential section through the nodular region of the outer segments shown in (c). Several examples of very closely appositioned elements are demonstrated (arrowed). The bar marker is $10 \mu \mathrm{m}$

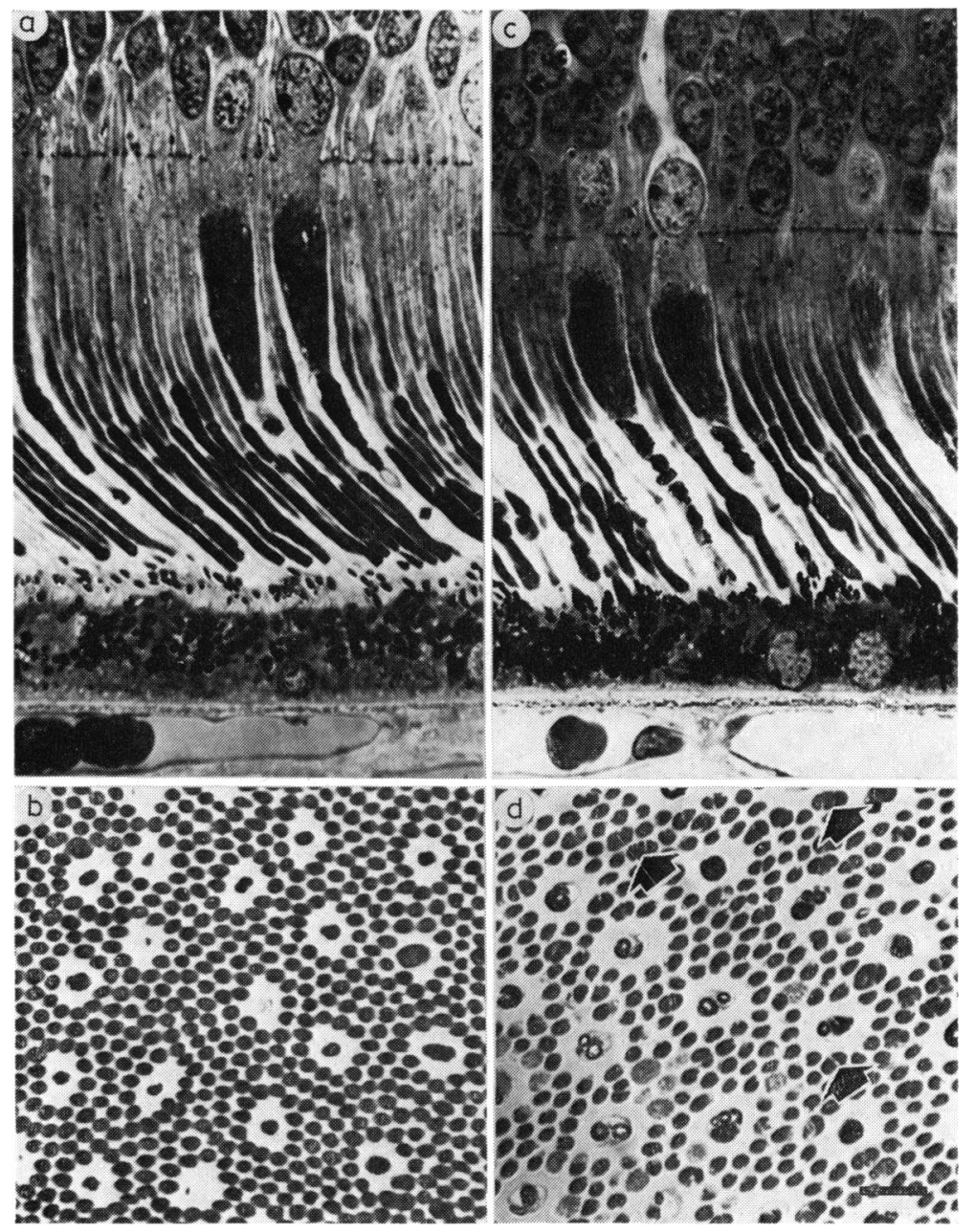

(Fig. 2). These corrugations occurred in two forms. In some rods the disc stack was displaced in a sinusoidal curve, which was compressed within the cells' boundary membrane. In other rods the whole outer segment including the boundary membrane was distorted into a series of curves. Both configurations resulted in localised corrugations in which the membranes in the ascending and descending limbs were closely appositioned. These convolutions increased the absolute length of the outer segments by 6 to $10 \mu \mathrm{m}$ and their disc content by 20 to $40 \%$. However, the axial distance between their cilium and the pigment epithelium remained in the same range as those of young eyes. These systems were never found to extend any distance into the receptor sheath systems of the apical membrane of the pigment epithelium. Within the sheaths small dislocations of the disc stacks were occasionally observed, and these were similar to those seen in young eyes.

When the convolutions were sectioned at rightangles to the long axis of the outer segments they appeared as a fused association of independent elements from several juxtapositioned cells (Fig. 2). The number of elements involved depended upon the number of convolutions experienced by a particular outer segment but ranged between 2 and 5 discs. The 2 modes of outer segment corrugation were clearly distinguishable, with groups of discs either being bounded by a single membrane system, or multiple boundary membranes with varying degrees of confluence. These sections also demonstrated that multiple convolutions were not confined to a single plane (Fig. 2). Similar changes have also been observed in the rod outer segments of a mature (10 to 15 years) rhesus monkey.

In human retinae in the present study inter- 


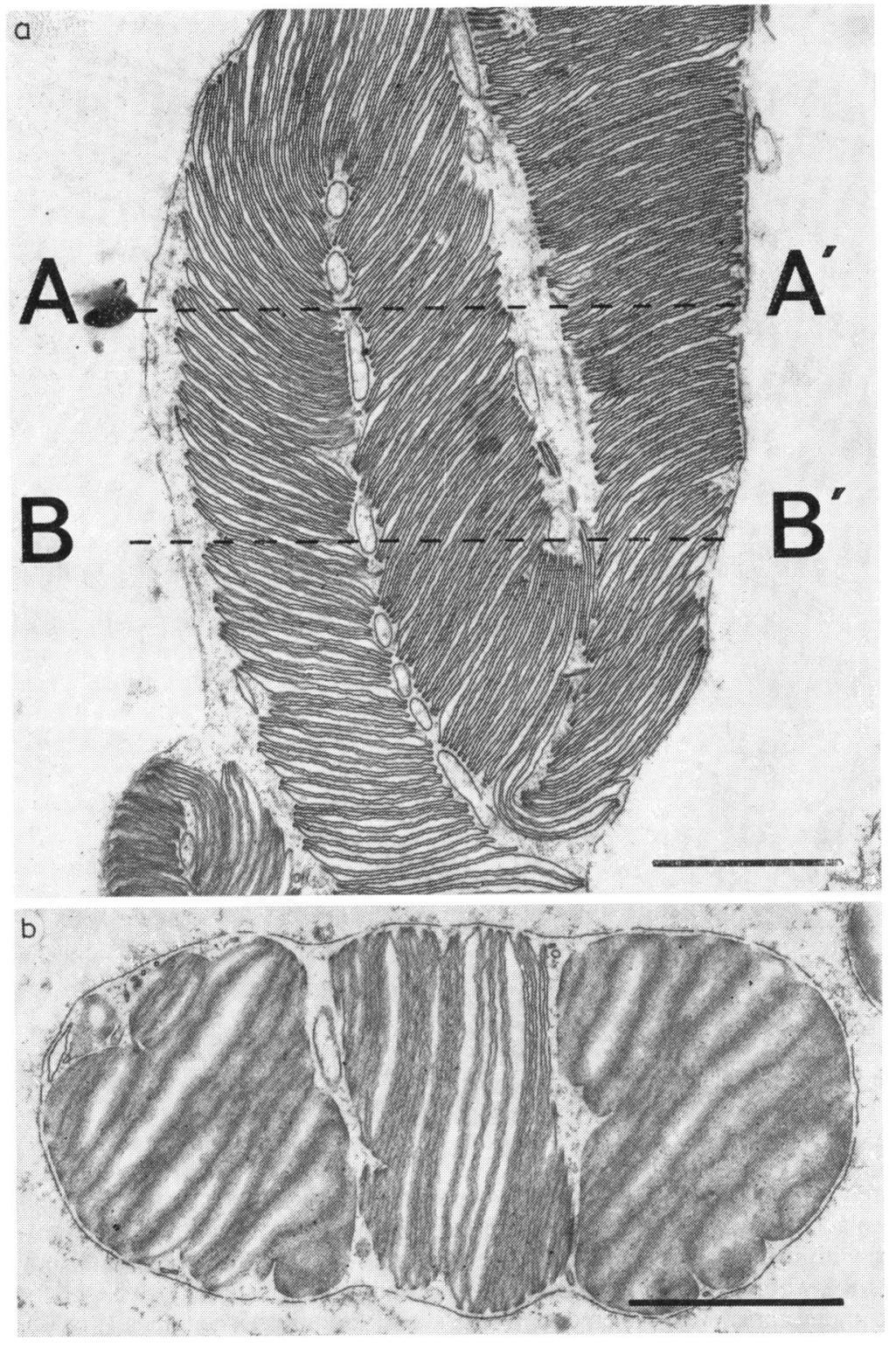

Fig. 2 (a) Electron micrograph of a transverse section through a rod outer segment nodule showing a convolution of the disc stack and varying degrees of membrane fusion between the ascending and descending limbs. Therefore a tangential section on plane $\mathrm{A}-\mathrm{A}^{\prime}$ would appear as a system of discs enclosed by a single boundary membrane as in (b), while a section on plane $\mathrm{B}-\mathrm{B}^{\prime}$ would appear as 3 discs each with their own boundary membranes. The bar marker is $1 \mu \mathrm{m}$

(b) An electron micrograph of a tangential section through a rod outer segment nodule as described in (a). The bar marker is $1 \mu \mathrm{m}$ receptor membrane associations were found between processes from the inner segments (Fig. 3). These were similar to those previously described in other animals (Fain et al., 1976). However, in all such associations the integrity of participating membranes was maintained, and no fusion of cells was observed.

A detailed account of the changes observed in the pigment epithelium and Brüch's membrane in the present survey forms the basis of a future report. However, certain of these changes may be pertinent to those seen in the rods and are therefore briefly mentioned. In all retinae examined electron-dense deposits of lipofuscin were observed within the pigment epithelial cells, and both electron and fluorescence microscopy confirmed previous findings that such deposits increase with age (Hogan et al., 1971). In young eyes lipofuscin inclusions were most abundant in epithelial cells in the macular and perimacular regions.

\section{Discussion}

Loss of order in the outer segments of photoreceptor cells occurs in response to both pathological 
Fig. 3 (a) Low-power electron micrograph of a tangential section through the inner segments of the photoreceptor cells in a 54-yearold woman. The interreceptor matrix is much reduced in this region of the retina but still apparent. The bar marker is $2 \mathrm{um}$.

(b) Electron micrograph of a similar section to that in (a) taken just external to the outer limiting membrane. Specialised junctional complexes may be seen both between appositioned rods (closed arrows) and between rods and cones (open arrows). The bar marker is $1 \mathrm{um}$
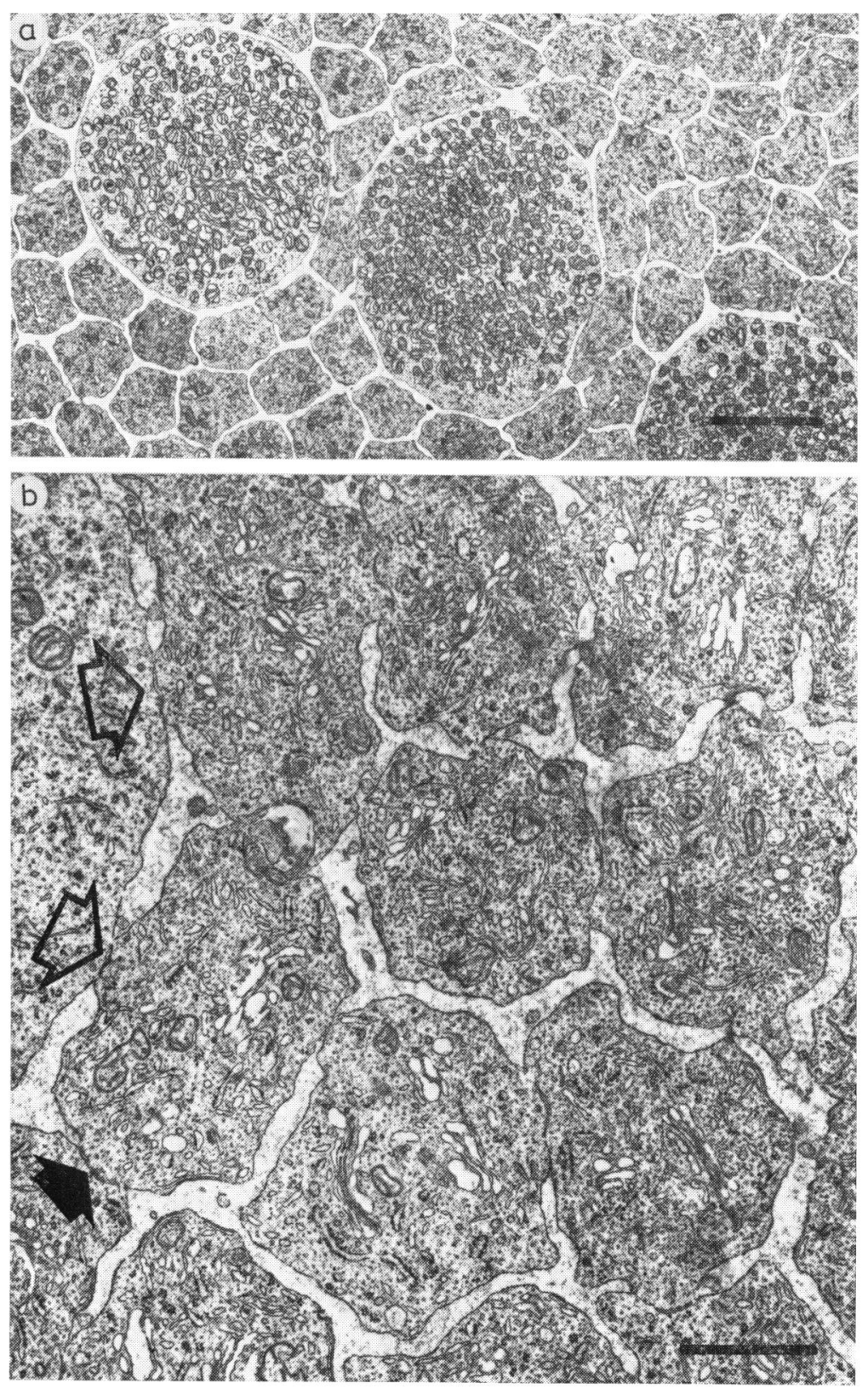

and histological processes. In vivo the receptors respond to a variety of disturbances in their microenvironment by failing to maintain the regular periodicity of their component disc membranes. After mild or transient injuries angular translocations of groups of discs occur within the disc stack. In episodes of severe or protracted in erference with receptor homoeostasis the disc membranes degenerate into vesicular or whorl-like configurations.
Typical examples of these degenerative changes are seen in such conditions as retinal detachment (Kroll and Machemer, 1968), vitamin deficiencies (Hayes, 1974), and thermal (Marshall, 1970a) or photic injury (Kuwabara, 1970). In each of these conditions the persistent changes in outer segment morphology occur as a result of fusion of membranes of both individual and juxtapositioned discs. This capacity for disc fusion is also seen in an extracellular 
environment and between discs originating from different receptor cells, as in the RCS rat discs shed from the rod tips, which are not phagocytosed by the pigment epithelium but come together to form huge sheets of lamellar material (Dowling and Sidman, 1962). Similar examples of disc membrane fusion are seen in vitro in experiments in which isolated rod outer segments are exposed to osmotic shock (Falk and Fatt, 1973) and in some histological techniques in which certain types of fixative induce such membrane fusion (Tormey, 1964). These observations imply that active processes are required for the continued maintenance of disc integrity. In contrast to disc membranes the boundary membranes of the outer segments of receptor cells remain discrete throughout each of these pathological processes and never fuse with those of neighbouring cells. The one exception to this is that portion of the boundary membrane shed from the tips of rods in the RCS rat.

In the present study areas of disorganisation arising from degenerative processes involving the discs were few in young eyes and consisted of the angular translocation of groups of discs within the boundary membranes of the rod outer segments. The vesicles seen within the plane of the discs resulted from section planes running either through the disc lobes or crenulations or through the fimbriae associated with the ciliary incisure (Pedler and Tilly, 1967). These latter incursions in the disc membrane do not maintain their stacking register on passing away from the cilium and usually revolve varying degrees in their axis of symmetry on their passage down the outer segment. Thus isolated or single multiple rows of vesicles with axial dimensions identical to those of the discs are observed throughout the length of the outer segments.

Changes in the appearance of receptor outer segments induced by histological processing are well known, and in all ophthalmic pathology laboratories the maintenance of the correct stereo spatial relationships between the neural retina and the pigment epithelium is a perennial problem. Many histological techniques result either in the detachment of the retina or in its compression against this underlying layer. In this latter group the receptor outer segments are either distorted to run at an acute angle towards the pigment epithelium, or they assume a sigmoid appearance. In affected samples all the receptors in any given area are similarly distorted. The convolutions that occur in the outer segments of older eyes in the present study (Fig. 2) are not an artefact resulting from the tissue processing techniques. They show a discontinuous distribution in histological sections of retina and an increasing incidence with age.
In many animals (Young, 1976) including monkey (Young, 1971), autoradiographic studies have shown that the discs in rod outer segments are in a state of flux. These experiments show that throughout life new discs are continuously formed in the region of the cilium, and are progressively displaced down the outer segment towards the pigment epithelium by successive disc production.

In monkeys the outer segment transit time for a disc is 9 to 13 days (Young, 1971). Old discs at the tips of the outer segments are removed by the phagocytic action of the pigment epithelium, a process which exists in dynamic equilibrium with that of disc production. Once inside the pigment epithelium the groups of engulfed discs, called phagosomes, undergo lysis, which induces their progressive degeneration (Marshall, 1970b; Ishikawa and Yamada, 1970). The degenerative end products of lysis are voided from the epithelial cells via Brüch's membrane into the choriocapillaris. From simple calculations it can be shown that in the monkey each epithelial cell destroys well over 1000 discs each day. This amounts to an enormous quantity of membranous material passing through the pigment epithelium during the life span of an animal.

This system has not been studied autoradiographically on human material, but substantive morphological evidence indicates its existence (Spitznas and Hogan, 1970). Morphological studies also suggest that the pigment epithelium in man is involved in the phagocytosis of cone outer segments (Steinberg et al., 1977). The observations of increasing deposits of lipofuscin within the pigment epithelium coupled with a progressive build-up of inclusions in Brüch's membrane in ageing human eyes have been interpreted as the products of incomplete breakdown or transport of such phagocytosed outer segment material (Hogan, 1972).

It has also been suggested that both of these inclusions are manifestations of a reduction in the ability of the pigment epithelium to contend with its phagocytic load and that their presence further disturbs the dynamic equilibrium of rod growth and phagocytosis. Such perturbations could arise from a progressively increasing impedance to the outflow of phagosomal debris induced by the successive deposition of such material within Brüch's membrane. This mechanism could therefore result either in a massive build-up of debris, with the production of drusen and possibly more serious senile lesions (Sarks, 1976) or in a decrease in the rate of phagocytosis.

If the rate of disc production remains relatively constant throughout life but the rate of phagocytosis decreases, then rods must increase in length. Such an imbalance could well result in the convolutions 
described in the present study. This suggestion is supported by two further findings. First, the initial occurrence and subsequent highest incidence of these convolutions is in the perimacula, an area in which retinal pigment epithelial cells have a large lipofuscin content, and secondly this is an area in which drusen are commonly observed (Gass, 1973).

The degree of membrane fusion between the various limbs of the convolutions could result in three different appearances in sequential crosssections of a single convolution (Fig. 2). Thus, where the boundary membranes of the limbs had not fused, 'isolated' elements were observed; where these membranes were confluent, the system appeared as 2 or more discs within a single membrane. And, finally, various intermediate fusions imparted an appearance similar to cytoplasmic bridges. This latter form resulted in the misinterpretation of these systems as the fused elements of appositioned receptor cells (Borwein et al., 1977). The present findings are a reinterpretation arising from examination of data in both laboratories.

The functional implications of convoluted rods and the effects of their increasing numbers with age are unclear. The increase in disc content and absolute outer segment length does not seem significant because their axial length remains relatively constant through life. The increase in size of the catchment area presented to incident light in affected receptors may be of greater consequence to rod function. However, the variations between individual receptors would render analysis extremely difficult.

It is our pleasure to thank the medical and nursing staffs of the following hospitals whose co-operation in gathering samples has been essential: Addenbrookes Hospital, Cambridge; Hillingdon Hospital, London; King's College Hospital, London; Manchester Royal Eye Hospital; Middlesex Hospital, London; Moorfields Eye Hospital, London; and Victoria Hospital, London, Ontario. We are grateful to Miss E. Clarke and Mrs A. Karkahanis for technical assistance, and to both the Garrick Charitable Settlement and the Wellcome Trust for donations of scientific apparatus. We also acknowledge the US Army Medical Research and Development Command, and the Advisory Group for Aerospace Research and Development (AGARD) of NATO, for financial support.

\section{References}

Borwein, B., Medeiros, J. A., and McGowan, J. W. (1977). Fusing human rod outer segments from an eye enucleated for choroidal melanoma. Investigative Ophthalmology, 16, 678-683.

Dowling, J. E., and Sidman, R. L. (1962). Inherited retinal dystrophy in the rat. Journal of Cell Biology, 14, 73-109.

Fain, G. L., Gold, G. H., and Dowling, J. E. (1976). Receptor coupling in the toad retina. Cold Spring Harbour Symposia on Quantitative Biology, 40, 547-561.

Falk, G., and Fatt, P. (1973). Changes in structure of the disks of retinal rods in hypotonic solutions. Journal of Cell Science, 13, 787-799.

Gass, J. D. M. (1973). Drusen and disciform degeneration, macular detachment and degeneration. Archives of Ophthalmology, 90, 206-217.

Hayes, K. C. (1974). Retinal degeneration in monkeys induced by deficiencies of vitamin E or A. Investigative Ophthalmology, 13, 499-510.

Hogan, M. J. (1972). Role of the retinal pigment epithelium in macular disease. Transactions of the American Academy of Ophthalmology and Otolaryngology, 76, 64-80.

Hogan, M. J., Alvarado, J. A., and Weddell, J. E. (1971). Histology of the Human Eye: An Atlas and Textbook. W. B. Saunders: Philadelphia.

Huber, J. D., Parker, F., and Odland, G. F. (1968). A basic fuchsin and alkalinized methylene blue rapid stain for epoxy-embedded tissue. Stain Technology, 43, 83-87.

Ishikawa, T., and Yamada, E. (1970). The degradation of the photoreceptor outer segment within the pigment epithelial cell of rat retina. Journal of Electron Microscopy, 19, 85-91.

Kroll, A. J., and Machemer, R. (1968). Experimental retinal detachment in the owl monkey: III. Electron microscopy of retina and pigment epithelium. American Journal of Ophthalmology, 66, 410-427.

Kuwabara, T. (1970). Retinal recovery from exposure to light. American Journal of Ophthalmology, 70, 187-198.

Laties, A. M., and Enoch, J. M. (1977). An analysis of retinal receptor orientation. I. Angular relationship of neighbouring photoreceptors. Investigative Ophthalmology, 10, 69-77.

Marshall, J. (1970a). Thermal and mechanical mechanisms in laser damage to the retina. Investigative Ophthalmology, 9, 97-115.

Marshall, J. (1970b). Acid phosphatase activity in the retinal pigment epithelium. Vision Research, 10, 821-824.

Meek, G. (1963). In discussion of 'A scheme for section staining in electron microscopy', by Mercer, E. M. Journal of the Royal Microscopical Society, 81, 184.

Pedler, C. M. H., and Tilly, R. (1967). The fine structure of photoreceptor discs. Vision Research, 7, 829-836.

Sarks, S. H. (1976). Ageing and degeneration in the macular region: a clinico-pathological study. British Journal of Ophthalmology, 60, 324-341.

Spitznas, M., and Hogan, M. J. (1970). Outer segments of photoreceptors and the pigment epithelium: interrelationship in the human eye. Archives of Ophthalmology, 84, 810-819.

Steinberg, R. H., Wood, I., and Hogan, M. J. (1977). Pigment epithelial ensheathment and phagocytosis of extra foveal cones in human retina. Philosophical Transaction of the Royal Society of London, Series B, 277, 459-474.

Tormey, J. McD. (1964). Differences in membrane configuration between osmium tetroxide-fixed and glutaraldehyde-fixed ciliary epithelium. Journal of Cell Biology, 23, 658-664.

Young, R. W. (1971). Shedding of discs from outer segments in the rhesus monkey. Journal of Ultrastructure Research, 34, 190-203.

Young, R. W. (1976). Visual cells and the concept of renewal. Investigative Ophthalmology, 15, 700-725. 